\title{
Facile and Ultraclean Graphene-on-Glass Nanopores by Controlled Electrochemical Etching
}

\author{
Xiaoyan Zhang, Pauline M. G. van Deursen, Wangyang Fu,* and Grégory F. Schneider*
}

Cite This: ACS Sens. 2020, 5, 2317-2325

Read Online
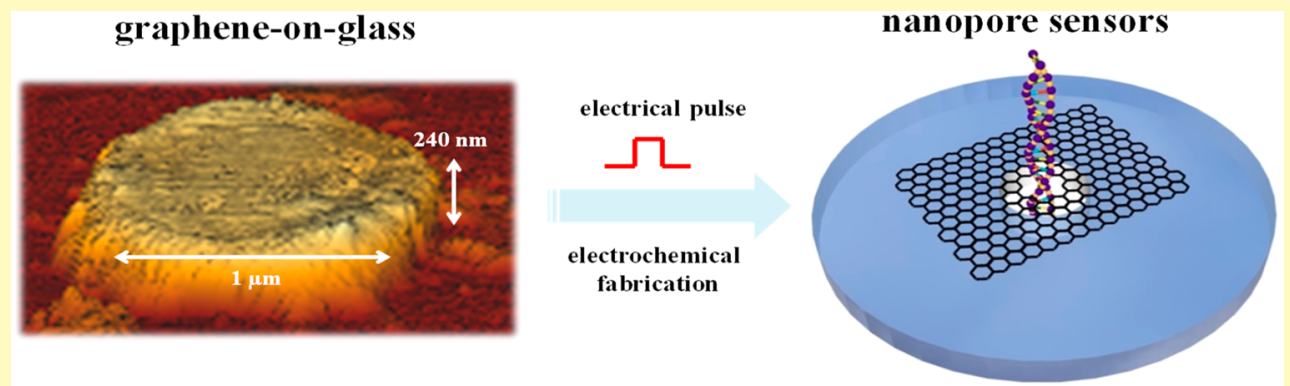

ABSTRACT: A wide range of approaches have been explored to meet the challenges of graphene nanostructure fabrication, all requiring complex and high-end nanofabrication platform and suffering from surface contaminations, potentially giving electrical noise and increasing the thickness of the atomically thin graphene membrane. Here, with the use of an electrical pulse on a lowcapacitance graphene-on-glass (GOG) membrane, we fabricated clean graphene nanopores on commercially available glass substrates with exceptionally low electrical noise. In situ liquid AFM studies and electrochemical measurements revealed that both graphene nanopore nucleation and growth stem from the electrochemical attack on carbon atoms at defect sites, ensuring the creation of a graphene nanopore. Strikingly, compared to conventional TEM drilled graphene nanopores on SiN supporting membranes, GOG nanopores featured an order-of-magnitude reduced broadband noise, which we ascribed to the electrochemical refreshing of graphene nanopore on mechanically stable glass chips with negligible parasitic capacitance $(\sim 1 \mathrm{pF})$. Further experiments on double-stranded DNA translocations demonstrated a greatly reduced current noise, and also confirmed the activation of single nanopores. Therefore, the exceptionally low noise and ease of fabrication will facilitate the understanding of the fundamental property and the application of such atomically thin nanopore sensors.

KEYWORDS: graphene nanopores, ultraclean, low noise, electrochemical etching, glass substrate

$\mathrm{O}$ wing to the atomically thin and ion-impermeable structure of graphene, nanopores ${ }^{1-3}$ and nanogaps ${ }^{4}$ in graphene provide the opportunity for reaching single building block resolution in biomolecule sequencing. ${ }^{5}$ In nanopore sequencing, a strand of DNA traverses a nanopore submerged in a saline recording solution. The ionic current through the pore is monitored as the DNA strand passes, providing information on the local diameter of the strand. From the standpoint of device fabrication, the challenge lies in the reliable formation of atomic clean and stable pores or gaps in graphene at the nanometer scale. ${ }^{6}$ Among the current methodologies, transmission electron microscopy (TEM) sculpting with a highly focused electron beam is the most controllable. ${ }^{7}$ Nevertheless, the high cost and potential carbon contamination of the TEM technique make it unsuitable for scaling up the production of nanopore or nanogap devices. In addition, the TEM processing step limits the choice of substrates, as TEM substrates need to be compatible with a TEM holder and electrically conductive-to prevent charge accumulation during exposure to the electron beam. Free- standing thin silicon-nitride ( $\mathrm{SiN}$ ) membranes (several tens of nanometers in thickness supported on Si substrates) with a prefabricated nanopore (several hundred of nanometers in diameter) are commonly used as supporting substrates for fabricating graphene nanopores by TEM., ${ }^{2,3}$ The presence of a Si chip, however, raises a drawback during the nanopore measurement when submerged in the saline solution: the large parasitic capacitance $(\gg 10-100 \mathrm{pF})$ resulting from the relatively thin supporting $\mathrm{SiN}$ membrane between the semiconducting $\mathrm{Si}$ and the saline solution induces high current noise. ${ }^{8,9}$ Recently, electrical pulse fabrication was used as an alternative route to efficiently and reproducibly fabricate graphene nanopores. ${ }^{10}$ However, so far such graphene

Received: April 30, 2020

Accepted: June 23, 2020

Published: June 23, 2020 

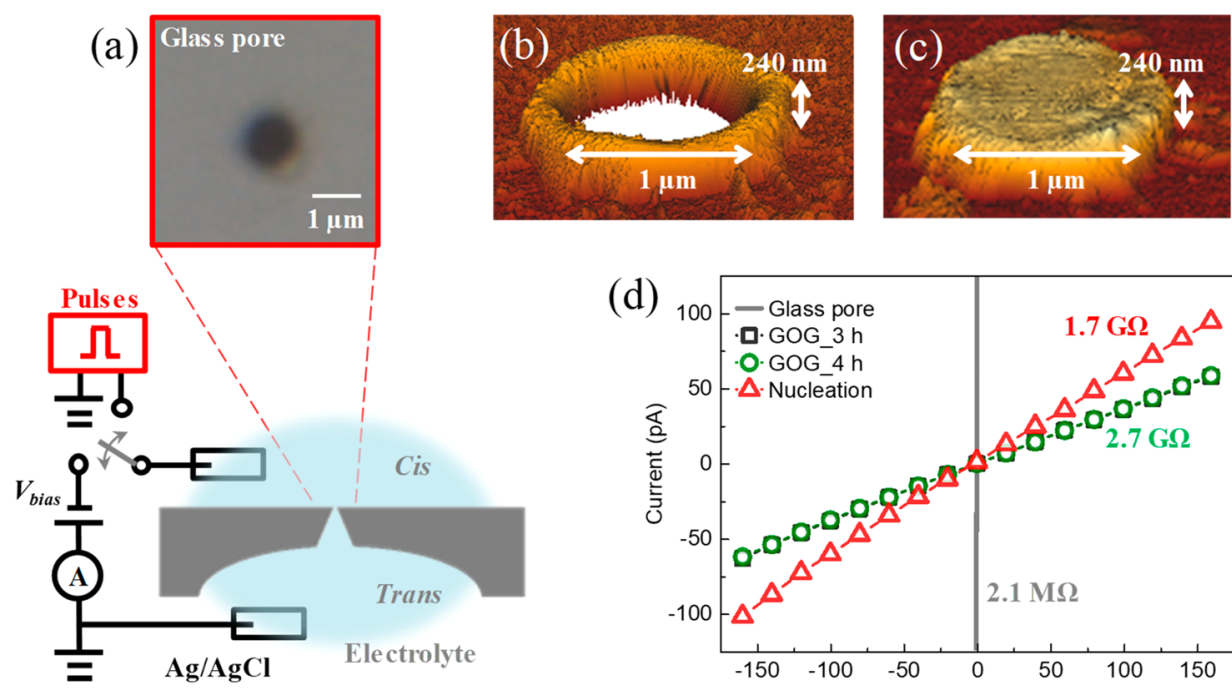

(d)

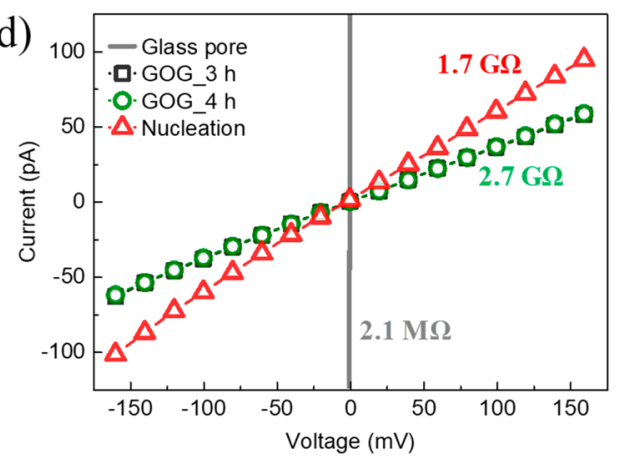

Figure 1. Preparation and characterization of the graphene-on-glass (GOG) substrate. (a) Upper panel: an optical image shows a well-defined aperture at the center of a glass chip; lower panel: experimental scheme consisting of "cis" and "trans" fluidic chambers with a glass pore between the two reservoirs. The $\mathrm{Ag} / \mathrm{AgCl}$ reference electrode residing in the cis fluidic chamber was used to introduce transmembrane voltage pulses for the fabrication of GOG nanopores. (b) AFM image of a clean, smooth-rimmed glass pore over a $1 \mu \mathrm{m}$-diameter circular aperture. (c) AFM image of a glass pore completely covered by a free-standing CVD graphene, forming the GOG substrate. (d) Current-voltage curves of a bare glass pore (gray line) and a GOG substrate with a stable resistance of $2.7 \mathrm{G} \Omega$ (black rectangular and green circles) in $1 \mathrm{M} \mathrm{KCl}, 10 \mathrm{mM} \mathrm{Tris,} \mathrm{and} 1 \mathrm{mM}$ EDTA, pH 8.1. The corresponding increase in current (reduction in resistance, $1.7 \mathrm{G} \Omega$, red triangle) suggests nanopore nucleation after applying short-term $(50 \mathrm{~ms})$, high-voltage $(7 \mathrm{~V})$ pulses.

nanopores were fabricated on silicon-based semiconducting substrates with relatively high current noise and low bandwidth. Particularly, to a large extent, the mechanism of nanopore formation in graphene with the electrical pulse method, as well as the ultraclean nature of the nanopores, are unclear and therefore limiting the broad application of the electrical pulse approach. ${ }^{10}$

In this paper, we use low-capacitance, commercially available glass chips featuring a micrometer-sized aperture at the center and use it as a support for graphene. This graphene-on-glass (GOG) platform, as we called it, is then subjected to electrically pulsed voltages yielding the formation of ultraclean graphene nanopores. In contrast to the dielectric breakdown fabrication on silicon substrates, we could probe into electrochemical mechanism using in situ liquid AFM, where a graphene nanostructure could be enlarged by removing carbon atoms at its freestanding edges during the breakdown process. Further experiments on double-stranded DNA translocations not only confirmed the formation of a single GOG nanopore, but also demonstrated greatly reduced current noise which we ascribed to the electrochemical refreshing of graphene nanopore on mechanically stable glass chips with minimized parasitic capacitance.

\section{EXPERIMENTAL METHOD}

CVD Graphene Transfer and Liquid Handling. Polycrystalline CVD graphene was obtained from Graphenea with good monolayer homogeneity (>98\%) and micrometer-sized grains. Raman spectroscopy and imaging were performed on a WITec confocal spectrometer with a $100 \times$ objective (lateral resolution of $\sim 300 \mathrm{~nm}$ ) and blue laser $(488 \mathrm{~nm}, 1 \mathrm{~mW})$, confirming the relatively high quality of graphene on copper foils (not shown). Prior to CVD graphene transfer, the Nano-Patch-Clamp chips (Nanion) were subjected to sonication in deionized (DI) water and ethanol, followed by blow drying with nitrogen. The glass chips were then treated in oxygen plasma for 2 min. A capacitively coupled plasma system with a radiofrequency
(RF) of $40 \mathrm{kHz}$ and $200 \mathrm{~W}$ power from Diener electronic (Femto) was employed at room temperature with a base pressure below 0.02 mbar and a power of $50 \mathrm{~W}$ at a pressure of 2.4 mbar for oxygen plasma. A PMMA supported CVD graphene layer floating on DI water (obtained by spin coating PMMA, copper etching, and rinsing with DI water) was then transferred onto those glass chips. The PMMA layer was then dissolved in acetone in the last step of transfer, leaving freestanding CVD graphene on the glass chips with micrometer-sized conical-shaped aperture at the center (Figure 1). We adopted a protocol to use $50 \%$ ethanol (in DI water), DI water, and $1 \mathrm{M} \mathrm{KCl} / 10 \mathrm{mM}$ Tris solution $(\sim 11 \mathrm{~S} / \mathrm{m}$ at room temperature $)$ at $\mathrm{pH}$ 8.1. Such protocol allows complete wetting and electrical contact via $\mathrm{Ag} / \mathrm{AgCl}$ electrodes to both sides of the graphene membrane, as any air bubbles will be repelled because of the low surface tension of the $50 \%$ ethanol solution. The leakage conductance $(0.5-1 \mathrm{nS})$ can be attributed to ion transport through defect structures in the free-standing graphene and the gaps between graphene and glass substrate, ${ }^{1}$ which is negligible compared to pore conductance for pores larger than $1 \mathrm{~nm}(>6 \mathrm{nS}) .{ }^{10}$ The whole device is very stable: control experiment without electrical pulse shows almost no change of leakage current during the measurements.

AFM Microscopy. AFM images were performed on a JPK NanoWizard Ultra Speed machine. A silicon probe (AC240TS, Asylum Research) with $\sim 70 \mathrm{kHz}$ nominal resonance frequency in air and $\sim 30 \mathrm{kHz}$ resonance frequency in liquid was used. The images were scanned in intermittent contact mode, which allows one to work with soft and easily damaged material, in liquid and at room temperature with $512 \times 512$ pixels. Such collected images were then processed using a JPK SPM Data Processing software. The in situ liquid AFM images in the inside were performed both before and after the application of electrical pulses without drying the sample. Detailed pictures and illustrations of the in situ liquid AFM setup can be found in Figure S1.

Electrical Measurements and Pulse Generator. All the nanopore measurements were performed with an ultralow-noise patch clamp amplifier (Axopatch 200B, Molecular Devices) with resistive-feedback circuitry in whole-cell mode. $\mathrm{Ag} / \mathrm{AgCl}$ electrodes are used to detect ionic currents and to apply electric fields. An Agilent pulse generator (81101A) was used to apply 50-ms-long 
(a)

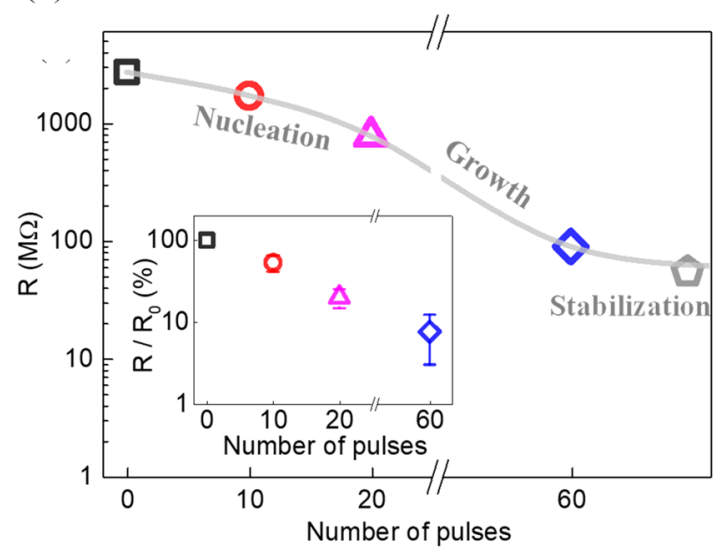

(b)
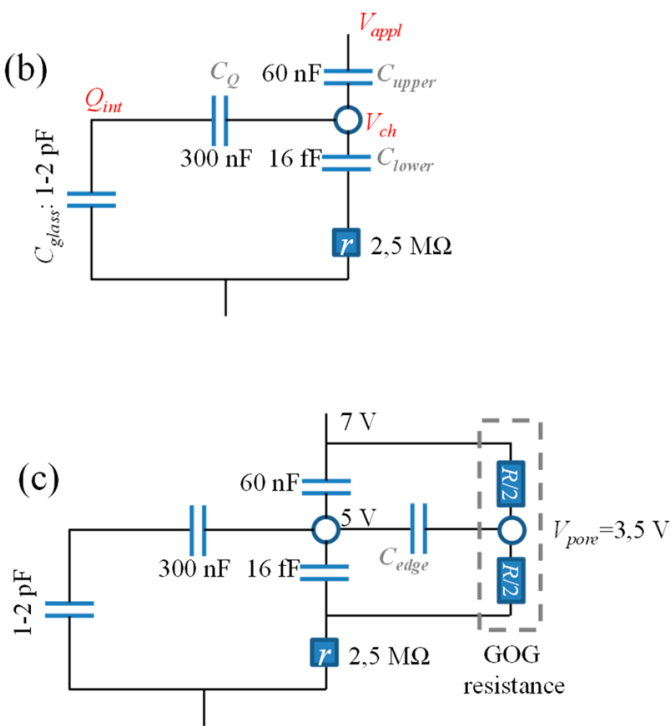
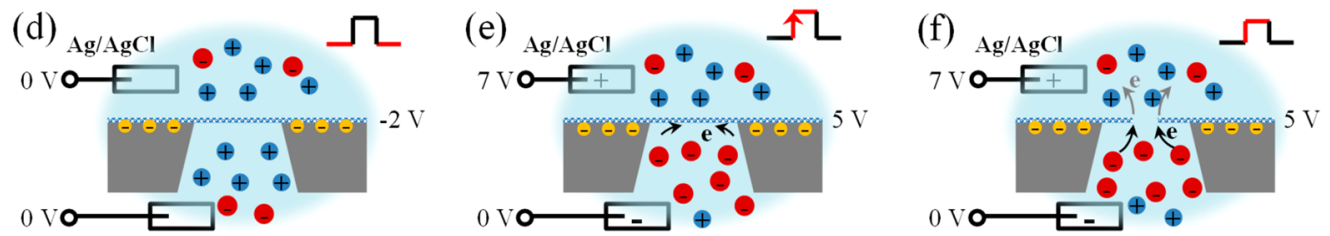

Figure 2. Mechanisms of GOG nanopore nucleation and formation by electrochemical etching. (a) Evolution of experimental GOG resistance $R$ vs number of $7 \mathrm{~V} / 50 \mathrm{~ms}$ pulses during electrical fabrication. Modest voltage pulses ( $5 \mathrm{~V} / 50 \mathrm{~ms}$ for 50 times) stabilize the $R$ from $88 \mathrm{M} \Omega$ (blue dot) to its final value at $54 \mathrm{M} \Omega$ (gray dot). Inset: average relative resistance $R / R_{0}$ vs number of pulses for three GOG nanopores, which exhibit similar resistance of $\sim 90 \mathrm{M} \Omega$ after experiencing 60 times of $7 \mathrm{~V} / 50 \mathrm{~ms}$ pulses. (b) Circuit diagram of the GOG system during nucleation. Any applied potential will mainly drop at the graphene/liquid interface inside the glass pore with the smallest capacitance $\left(C_{\text {lower }}\right)$ in the system. (c) Circuit diagram of the GOG system during nanopore formation. The nucleation of the graphene nanopore introduces a possible pathway for the current. As a result, (1) the potential of the electrolyte close to the graphene edge plane will be half of the applied potential: $3.5 \mathrm{~V}$; (2) a potential drop of $\sim 1.5 \mathrm{~V}=5 \mathrm{~V}-3.5 \mathrm{~V}$ will be developed across the graphene edges. (d) Scheme of the GOG system when both the "cis" and the "trans" chambers are grounded. (e) and (f) are the schemes of the GOG platform during nanopore nucleation and growth under voltage pulses of $7 \mathrm{~V}$, respectively.

voltage pulses. To protect the amplifier from large input voltages, a mechanical switcher was used to disconnect the amplifier before each pulse. The Axopatch 200B amplifier was reconnected $2 \mathrm{~s}$ after each pulse such that the GOG system gets stabilized. We empirically adopted a $7 \mathrm{~V} / 5 \mathrm{~V}$ protocol for electrochemical etching as previous electrical pulse fabrication method also reported the lowest voltage to reliably nucleate pores $(\sim 7 \mathrm{~V})$ and the lowest voltage to reliably enlarge nucleated pores $(\sim 5 \mathrm{~V}):(1) 7 \mathrm{~V}$ pulses with $50 \mathrm{~ms}$ period were applied repeatedly for both nucleation and formation, and (2) 5 $\mathrm{V}$ pulses with $50 \mathrm{~ms}$ were used for enlarging and stabilizing the GOG nanopores.

DNA Translocation and Noise Measurement. $\lambda$-DNA $(48,502$ base pairs, Lot: 1651504, from New England Biolabs Tech. N3011S) solution was prepared at a concentration of $15 \mu \mathrm{g} \mathrm{mL}^{-1}$ in $1 \mathrm{M} \mathrm{KCl}$ with $10 \mathrm{mM}$ Tris and $1 \mathrm{mM}$ EDTA, $\mathrm{pH}$ 8.1. DNA translocation events were recorded under 100 and $200 \mathrm{mV}$ bias voltage using a band-pass filter of $100 \mathrm{kHz}$ and a sampling rate of $500 \mathrm{kHz}$. The current traces were filtered using an 8-pole Bessel filter at $10 \mathrm{kHz}$ in Clampfit. A low noise data acquisition system (Axon Digidata 1550A, Molecular Devices) was adopted to further eliminate local noise and to record the translocation event signals. Power spectral densities were calculated from the Fourier transform of the autocorrelation function and dividing it by the sampling frequency and the sample length. For normalization, the power spectral densities were divided by the mean current of the corresponding traces. All of the analyses and fitting were done with a Matlab GUI-based package named Transalyzer. $^{29}$

\section{RESULTS AND DISCUSSION}

As the starting substrate, commercially available glass chips (insulating borosilicate Nano-Patch-Clamp chips purchased from Nanion) were used, containing a micrometer-sized conical aperture at the center (Figure la, upper panel). A monolayer of chemical vapor deposition (CVD) graphene was transferred onto the conical glass pore using the standard poly(methyl methacrylate) (PMMA) transfer method ${ }^{11}$ to obtain a so-called GOG membrane. This platform was submerged in a saline solution $(1 \mathrm{M} \mathrm{KCl}$ with $10 \mathrm{mM}$ Tris and $1 \mathrm{mM}$ EDTA, $\mathrm{pH} 8.1$ ) and connected on both side of the pore to an amplifier through $\mathrm{Ag} / \mathrm{AgCl}$ electrodes (Figure $1 \mathrm{a}$, lower panel). We measured the pore resistance, capacitance, and current noise spectra. Before the transfer of graphene, the $100-\mu \mathrm{m}$-thick insulating glass substrate contributes $1.8 \mathrm{pF}$ $\left(C_{\text {glass }}=1.8 \mathrm{pF}\right.$, Figure S2) to the total input capacitance with an aperture resistance of $2.1 \mathrm{M} \Omega$ (Figure $1 \mathrm{~d}$, gray line). Such small parasitic capacitance benefits low noise/large bandwidth sensing applications as discussed later. The as-prepared GOG ensemble exhibited a stable transmembrane resistance of 2.7 G $\Omega$ (black rectangles and green circles, Figure 1d), indicating a complete graphene coverage of the glass pore. AFM images (Figure $1 \mathrm{~b}$ and $\mathrm{c}$ ) show the morphology of the micrometersized glass pore with a clean, smooth-rimmed opening, before and after graphene transfer. In the 90 GOG substrates fabricated and tested, about 30 devices revealed finite 
transmembrane resistance in the order of $100 \mathrm{M} \Omega$ after transfer, attributed to inherent defects in CVD-grown graphene or electrostatic discharge effects ${ }^{12}$ (which we will discuss later). Frequently, significant capillary force leads to damaged graphene membranes with resistance $<10 \mathrm{M} \Omega$. After excluding samples with damaged graphene, the yield of GOG samples for subsequent processing was 31 devices (34\%) exhibiting transmembrane resistance of $>1 \mathrm{G} \Omega$.

In situ, a voltage was applied to open up a graphene nanopore. We applied short, high-voltage pulses $(50 \mathrm{~ms}, 7 \mathrm{~V})$ across the graphene membrane. Such a millisecond pulse is easier to achieve than the previously reported $250 \mathrm{~ns}$ pulses applied for graphene nanopore fabrication. ${ }^{10}$ In Figure 1d, repeated application of high voltage pulses $(50 \mathrm{~ms}, 7 \mathrm{~V}, 10$ repetitions with $2 \mathrm{~s}$ separation) lead to a decrease in the resistance to $1.7 \mathrm{G} \Omega$ (red triangles). In the following, we always use $50 \mathrm{~ms}$ pulses with $2 \mathrm{~s}$ separation time, if not otherwise specified.

We formed a GOG nanopore with a resistance of $88 \mathrm{M} \Omega$ when continuously repeating the $50 \mathrm{~ms}$ pulses for another 50 times after the first opening of the pore (Figure 2a, blue dot). To evaluate the controllability of the electrical fabrication, we averaged the relative resistance $R / R_{0}$ of three different GOG nanopores, which exhibited a similar resistance of around 90 $\mathrm{M} \Omega$ after experiencing 60 times $7 \mathrm{~V} / 50 \mathrm{~ms}$ pulses. As shown in the inset of Figure 2a, after applying $7 \mathrm{~V} / 50 \mathrm{~ms}$ pulses for 10, 20 , and 60 times, the standard deviations of $R / R_{0}:\left(\frac{\text { errorbar }}{R / R_{0}}\right)$ are $22 \%, 27 \%$, and $60 \%$, respectively, suggesting a reasonable reproducibility of our electrical fabrication method. In the final step, we kept enlarging and stabilizing the nanopore by using modest voltage pulses until a resistance of $54 \mathrm{M} \Omega$ was reached (Figure 2a, gray dot). This resistance value of $54 \mathrm{M} \Omega$ corresponds to a graphene nanopore with an equivalent $2.3 \mathrm{~nm}$ pore diameter assuming a regular round shape. ${ }^{1,2}$ The fact that this resistance value is kept unchanged over a time period of at least $1 \mathrm{~h}$ (Figure S3) suggests that the GOG nanopore is stable.

In the case of dielectric breakdown fabrication in $\mathrm{SiN}$ membranes, ${ }^{13}$ the applied electric fields (up to $\sim 1 \mathrm{~V} \mathrm{~nm}^{-1}$ ) are close to the dielectric breakdown strength of low-stress $\mathrm{SiN}$ films, and are able to induce accumulation of charge traps forming a highly localized conductive path. This results in the physical damage of the conductive path because of substantial power dissipation and heat generated, yielding a nanopore in the bulky SiN membrane. ${ }^{13}$ Unlike $10-30$-nm-thick SiN membranes, graphene is a conducting membrane with atomic thickness. Figure $2 \mathrm{~b}$ and Figure $\mathrm{S} 4$ represent the circuit diagram of the GOG and graphene on SiN systems, respectively. Instead of crossing the membrane, the potential drop $V_{\text {appl }}$ occurs mainly at the graphene/liquid interface in the glass pore where the charging capacitance is smallest: $C_{\text {lower }}=$ $16 \mathrm{fF} \ll C_{\text {glass }}=1-2 \mathrm{pF}, C_{\mathrm{Q}}=300 \mathrm{pF}, C_{\text {upper }}=60 \mathrm{pF}$, where $C_{\mathrm{Q}}$ and $C_{\text {upper }}$ represent the quantum capacitance and the interface capacitance at the upper side of the graphene membrane, respectively. To date, little is known about the mechanism that is responsible for graphene nanopore nucleation and growth.

As a rough estimation, we calculated the negatively charged surface group density at the graphene/glass interface $Q_{\text {int }}$ to be $5 \times 10^{17} \mathrm{~m}^{-2}$ in a solution of $10 \mathrm{mM}$ Tris $(\mathrm{pH}=8.1$, see also the Supporting Information). ${ }^{14}$ Therefore, a potential drop $V_{\mathrm{ch}}$ $=V_{\text {vari }} \sim-4 \mathrm{~V}$ would be obtained upon submerging the GOG substrate in the saline solution (grounded, $V_{\text {appl }}=0 \mathrm{~V}$ ). On the other hand, a potential drop $V_{\text {ch }}$ at the graphene-electrolyte interface (inside the glass pore) can, in principle, cause various electrochemical processes with electron transfer from graphene to electrolyte. It is most likely that such electron transfer tends to stabilize $V_{\text {ch }}$ at $-2 \mathrm{~V}$ (Figure $2 \mathrm{~d}$ ), the potential barrier of $\mathrm{H}^{+}$ reduction at graphene cathode (considering the overvoltage). Compared to the fast electrostatic charging of the electric double layer at $C_{\text {lower }}(100 \mathrm{~ns}-1 \mu \mathrm{s}),{ }^{15,16}$ this discharging process (discharging time constant of $\tau=r C_{\text {upper }}=150 \mathrm{~ms}$ ) is relatively slow and is therefore presumably due to electron transfer from graphene to the electrolyte. The detailed mechanism on ultraclean graphene nanopore/nanogap formation via electrochemical etching is discussed in Supporting Information. In principle, the obtained graphene nanopore has a sharp nanopore edge without possible amorphous carbon contaminations, which holds the potential of ultimate atomic spatial resolution. In addition, our previous experiments ${ }^{17}$ have demonstrated greatly improved electronic performance of electrochemically cleaned CVD graphene biosensors compared to their as-fabricated counterparts, including suppressed lowfrequency $1 / f$ noise, yielding an increased signal-to-noise ratio (SNR).

Figure $2 \mathrm{e}$ illustrates the charge distribution across the graphene membrane during a voltage pulse. During the first $0.1-1 \mu$ s from the start of the $7 \mathrm{~V}$ voltage pulse, a voltage drop of $V_{\text {ch }}=5 \mathrm{~V}$ (instead of $7 \mathrm{~V}$, due to the offset voltage $-2 \mathrm{~V}$ in Figure $2 \mathrm{~d}$ ) develops across $C_{\text {lower }}$ (with the smallest capacitance of $16 \mathrm{fF}$ among all the capacitors in the system). The nucleation of the graphene nanopore is taking place under this $V_{\text {ch }}=5 \mathrm{~V}$ during the following $50 \mathrm{~ms}$ time period. Here, the voltage drop at $C_{\text {lower }}=5 \mathrm{~V}$ has significantly passed the potential barrier of $\mathrm{Cl}^{-} / \mathrm{Cl}_{2}$ and $\mathrm{OH}^{-} / \mathrm{O}_{2}$ oxidization reactions at graphene anode, which occur around $2 \mathrm{~V}$. Consequently, we expect the electrochemical formation of $\mathrm{Cl}_{2}, \mathrm{O}_{2}$, and more aggressively, $\mathrm{Cl} \cdot, \mathrm{O} \cdot$, and $\mathrm{O}_{3} \cdot$ radicals, ${ }^{18}$ which can attack carbon atoms in graphene-especially the ones at defect sites in the graphene crystal lattice-and are responsible for atomically sharp nanopore nucleation. Notably, the separation time of $2 \mathrm{~s}$ between pulses seems to be sufficient for dissolving any gases formed during the pulse period of $50 \mathrm{~ms}$ and stabilizing the system before subsequent pulses, as we did not observe any gas bubble forming even after up to 200 pulses. 7 $\mathrm{V} / 500 \mathrm{~ms}$ pulses, on the other hand, lead to immediate gas bubble formation. As soon as the applied voltage $V_{\text {appl }}$ drops from 7 to $0 \mathrm{~V}$, the actual potential $V_{\mathrm{ch}}$ drops to and stabilizes at $-2 \mathrm{~V}$ again (Figure $2 \mathrm{~d}$ ). Repeating this process up to $10-20$ times normally led to nanopore nucleation with noticeable reduction in the GOG resistance, signaling pore formation, as mentioned above (red triangles, Figure 1d).

The successful nucleation of a graphene nanopore $(7 \mathrm{~V}, 50$ $\mathrm{ms}, 10$ times) introduces a possible pathway of the current. In Figure $2 c$, given that the resistance of the graphene nanopore $(R>1 \mathrm{G} \Omega)$ is dominantly larger than the glass pore resistance $(r=2-3 \mathrm{M} \Omega)$, we expect that half of the applied voltage is distributed at the nanopore: $V_{\text {pore }}=3.5 \mathrm{~V}$. During the GOG nanopore growth, a voltage drop up to $1.5 \mathrm{~V}$ occurs across the edge of the graphene pore $\left(V_{\text {edge }}=V_{\text {ch }}-V_{\text {pore }}=5 \mathrm{~V}-3.5 \mathrm{~V}\right.$, Figure $2 \mathrm{f}$ ), which is sufficient to initiate the electrochemical etching and refreshing effect, as below.

CVD graphene (or even exfoliated graphene) contains defects, including vacancies and grain boundaries. These defective carbon atoms, as well as possible amorphous carbon 
(a)

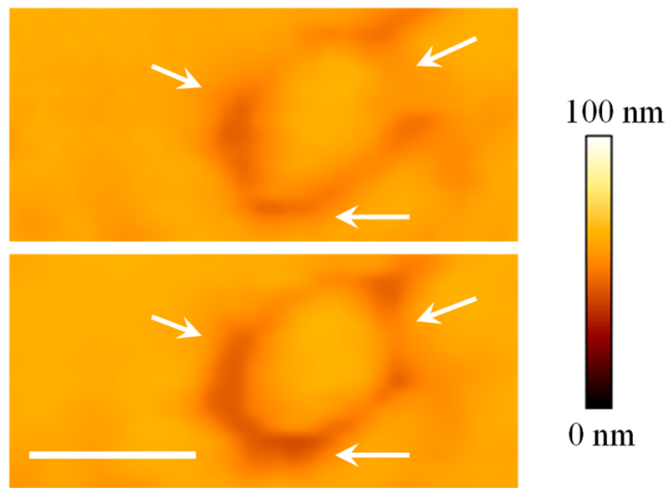

(b)

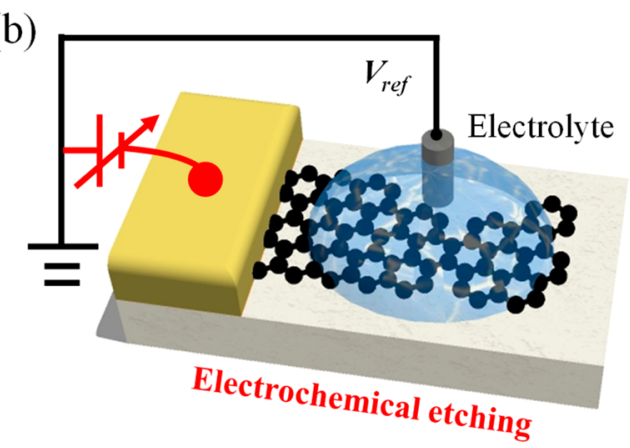

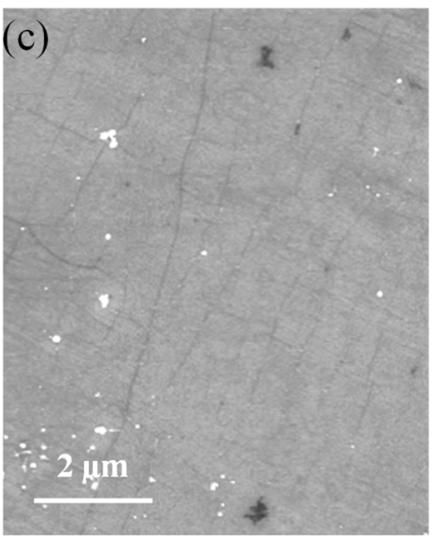
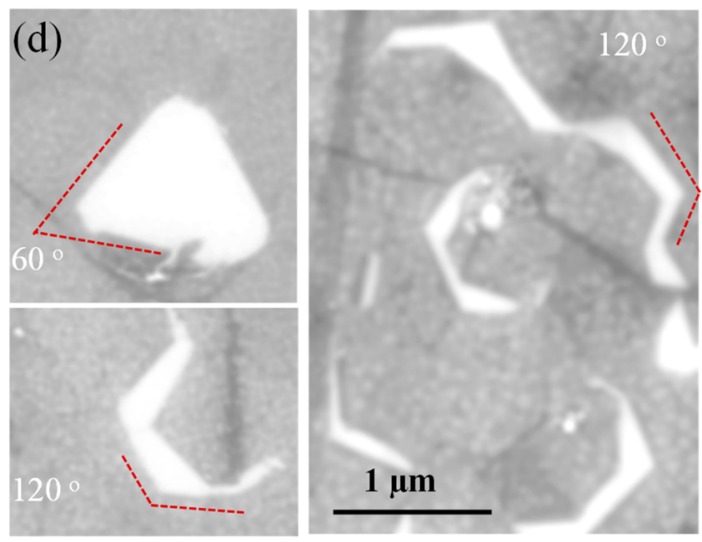

Figure 3. GOG nanopore formation via electrochemical etching. (a) In situ liquid AFM images scanned at the same position during graphene nanostructure enlargement under electric pulses, as indicated by the white arrows (upper panel: before the pulses; lower panel: after the pulses). Scale bar: $50 \mathrm{~nm}$. (b) Scheme of electrochemical etching of CVD graphene transferred on $\mathrm{Al}_{2} \mathrm{O}_{3}(20 \mathrm{~nm}) / \mathrm{SiO}_{2}(285 \mathrm{~nm}) / \mathrm{Si}$ substrate and the corresponding circuit diagram. (c) SEM image of as-transferred CVD graphene on the $\mathrm{Al}_{2} \mathrm{O}_{3} / \mathrm{SiO}_{2} / \mathrm{Si}$ substrate. (d) SEM images of the CVD graphene after $30 \mathrm{~min}$ of $1.6 \mathrm{~V}$ electrochemical etching in $1 \mathrm{M} \mathrm{KCl}$ solution. The exposed hexagonal edges of the CVD graphene (on the insulating $\mathrm{Al}_{2} \mathrm{O}_{3} / \mathrm{SiO}_{2} / \mathrm{Si}$ substrate in white color) suggest that the electrochemical etching process was most likely initiated from its grain boundaries contains active defects.

introduced during chip preparation and storage, are more inclined to be oxidized and attacked in an electrochemical reaction induced by the voltage drops at the graphene/liquid interface. Regrettably, given the extremely small amount of carbon atoms being removed from the graphene membrane during nucleation, it was impossible to directly study the reaction products to resolve the exact mechanisms of nanopore nucleation. However, using liquid AFM we were able to monitor the in situ electrochemical etching of graphene (Figure S4). We were able to trace the electrochemical etching of the graphene along its edge, after applying electrical pulses as in Figure 2a. Figure 3a (upper panel) shows the AFM image of an as-transferred GOG. This graphene area features exposed edges, represented by the darker yellow areas in Figure 3a, which can be ascribed to the mechanical damage and/or electrostatic discharge during the assembly of the GOG sample. As shown in the lower panel of Figure 3a, the electrical pulses attack the graphene edges (white arrows), evident from the widening of the darker regions. That is, defective carbon atoms at the exposed graphene edge are attacked, presumably due to the effective oxidation of carbon into reactive products, which dissolve into the electrolyte solution during the electrochemical etching process. ${ }^{19,20}$ Naturally, such a solution based in situ electrochemical process produces clean graphene nanopores due to the progressive etching compared to TEM sculpting approaches.

To further shed light on the mechanism of pore formation, we transferred CVD graphene on an $\mathrm{Al}_{2} \mathrm{O}_{3}(20 \mathrm{~nm}) / \mathrm{SiO}_{2}(285$ $\mathrm{nm}) / \mathrm{Si}$ substrate (Figure $3 \mathrm{c}$ ) and examined electrochemical etching behaviors under similar conditions during nanopore formation. For that, we used a semiconductive silicon substrate instead of an insulating glass substrate to facilitate SEM imaging. We also applied an additional 20-nm-thick atomic layer deposition (ALD) $\mathrm{Al}_{2} \mathrm{O}_{3}$ layer to block any possible leakage of current through the substrate. ${ }^{21}$ As shown in Figure S5 in the Supporting Information, on polycrystalline CVD graphene, the growth started from high-density seeds with hexagonal features (see Figure S5a). As these hexagonal graphene flakes grew larger laterally and meet each other at the edges, grain boundaries containing vacancy or topological defects formed. In Figure 3b, we applied direct current (DC) voltage to the graphene using evaporated gold electrodes or silver paste, to apply a mild voltage of $1.6 \mathrm{~V}$ at the graphene/ liquid interface via a droplet of $1 \mathrm{M} \mathrm{KCl}$ solution (grounded via a reference electrode). This electrochemical condition is similar to the electrical condition during the growth of the graphene nanopore in the previous section of the paper $(1.5 \mathrm{~V}$ across the edges of the graphene pore, see Figure $2 \mathrm{c}$ and $\mathrm{f}$ ). After $30 \mathrm{~min}$, we were able to etch the CVD graphene as 
(a)

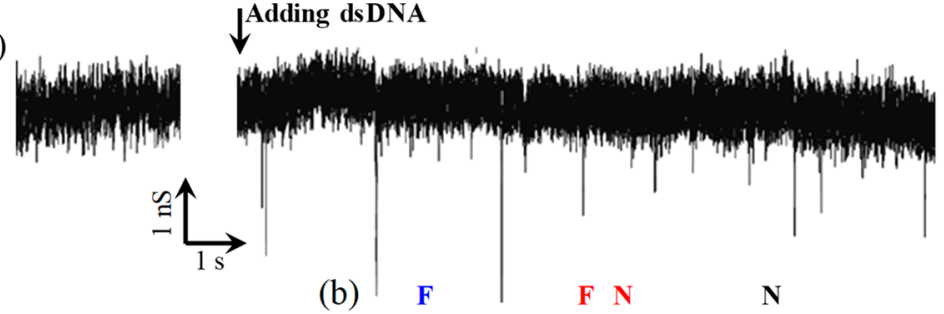

(b)

(c)
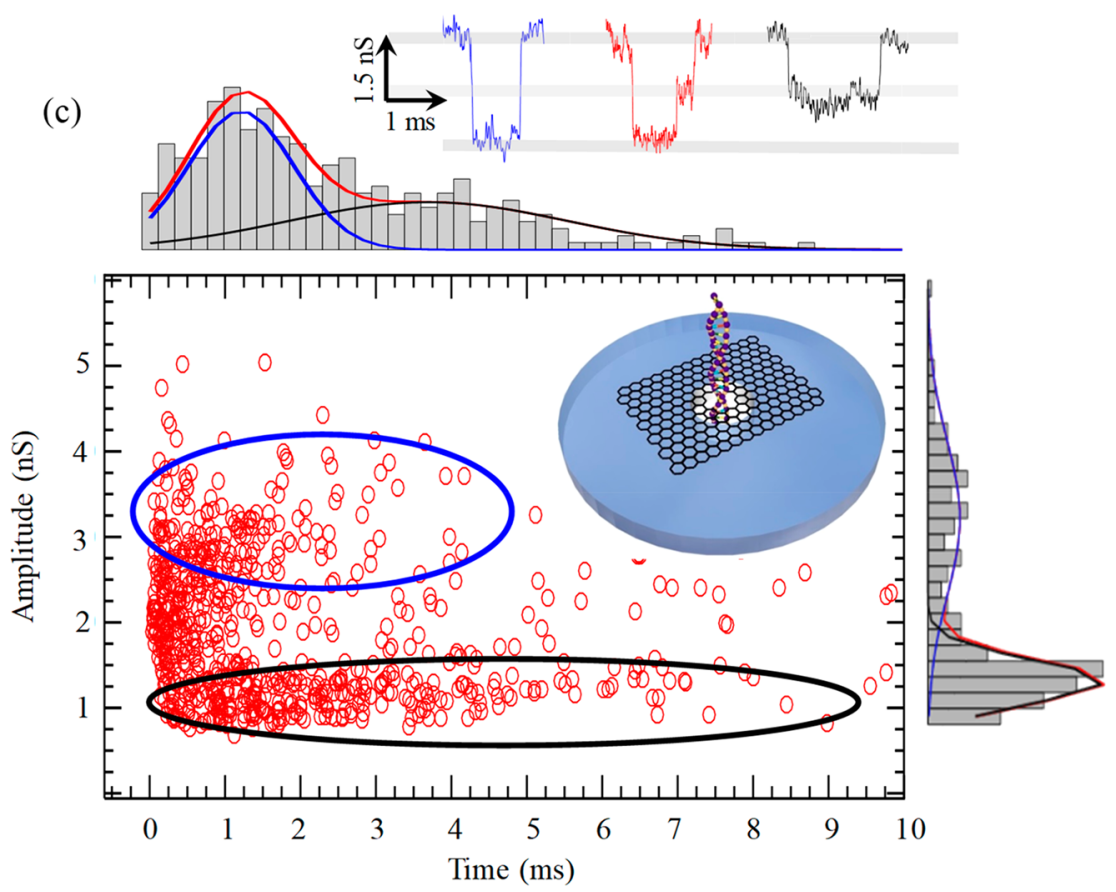

Figure 4. DNA translocations through a GOG nanopore. (a) Baseline conductance (left) and blockade events (right) upon addition of $48.5 \mathrm{~kb}$ double-stranded $\lambda$-DNA across a $16.5 \mathrm{~nm}(6 \mathrm{M} \Omega$ ) GOG nanopore. (b) Examples of translocation events of folded (F, blue), partially folded (F+N, red), and nonfolded (N, black) DNA molecules recorded at $200 \mathrm{mV}$. (c) Scatter diagram of the amplitude of the conductance blockade versus translocation time for $\lambda$-DNA translocate through the GOG nanopores. Inset is a schematic of a DNA molecule translocating through a GOG nanopore. The accompanying histograms for the DNA blockade time is included at the top of the Figure 4c, and the histograms for DNA blockade amplitude data is included on the right of Figure 4c, respectively. Regions of DNA translocation data for nonfolded and folded DNA translocation events are highlighted inside the black and blue circled areas, respectively. Each point in this scatter diagram corresponds to a single translocation event.

shown in Figure 3d. The exposed white-colored insulating $\mathrm{Al}_{2} \mathrm{O}_{3} / \mathrm{SiO}_{2} / \mathrm{Si}$ substrate reveals the hexagonal edges of the etched CVD graphene. The fact that the size as well as the hexagonal shape of the etched CVD graphene closely resemble those of its grain boundaries (see Figure S5) suggests that the electrochemical etching process was most likely initialized from and progressed along its grain boundaries, which contain active defects. It is at these defect sites that a graphene nanopore preferentially nucleats and grows (50 ms pulses of 5-7 V). Under electrical pulse condition, this electrochemical etching/ refreshing process forms an atomic sharp pore edge without contamination, which not only reduces the spatial resolution, but also was supposed as a source for high nanopore noise. ${ }^{22}$

To demonstrate the suitability of these GOG nanopores for single molecule measurements, we have performed DNA translocation experiments (Figure 4) after the electrical pulse fabrication of the GOG nanopore. DNA is negatively charged. Under an electric field, the molecules therefore move from anode $(-)$ to cathode $(+)$. When a graphene membrane with nanopore is placed between the two electrodes, the electric field concentrates across the nanopore and drags the DNA molecules from head-to-tail (including folded conformations) through the pore. Each nucleotide within the DNA strand could be read off when it is located at the narrowest constriction of the pore by detecting the ion current passing through the pore to probe the sequence of nucleotides constituting the DNA. We injected $\lambda$-DNA on the grounded side of the flow cell and applied a negative bias voltage to drive the negatively charged $\lambda$-DNA through the GOG nanopore with a $100 \mathrm{mV}$ or $200 \mathrm{mV} \mathrm{DC}$ voltage bias via $\mathrm{Ag} / \mathrm{AgCl}$ reference electrodes (Figure S6). Threading of the doublestranded $\lambda$-DNA molecule through the pore causes a transient reduction in the ionic current (conductance) measured with a low noise Axopatch amplifier (Figure 4a).

These translocation events can be distinguished based on the magnitude of the conductance blockade $(\Delta G)$. In Figure $4 \mathrm{~b}$, we observed two characteristic reductions in the ionic conductance $(\Delta G): 1.5 \mathrm{nS}$ and $3 \mathrm{nS}$, representing the nonfolded (N) and folded (F) translocation events, respectively. These $\Delta G$ values for $\lambda$-DNA in GOG pores are quite similar in magnitude to that previously measured for graphene pores with diameter of around $20 \mathrm{~nm}$ for the same conditions. ${ }^{2}$ We measured DNA translocations on 9 graphene nanopores with pore diameters ranging from 10 to $25 \mathrm{~nm}$, and 
(a)

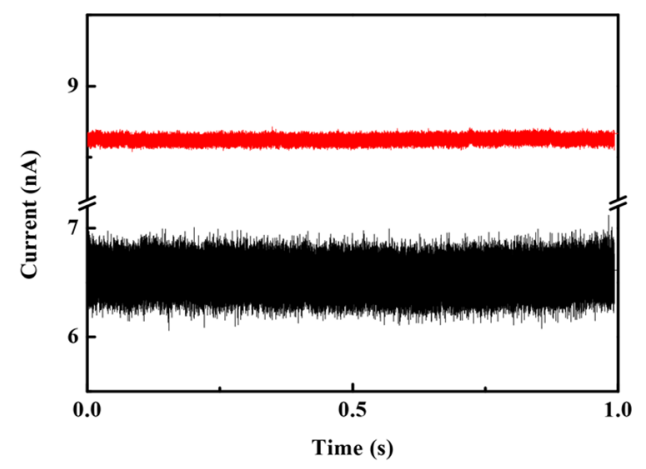

(c)

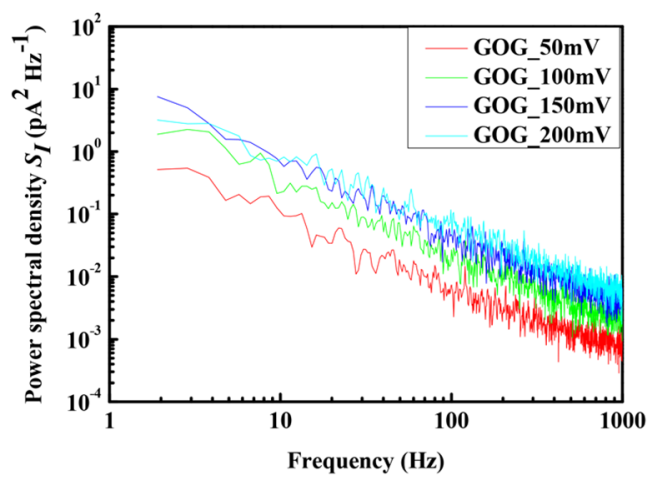

(e)

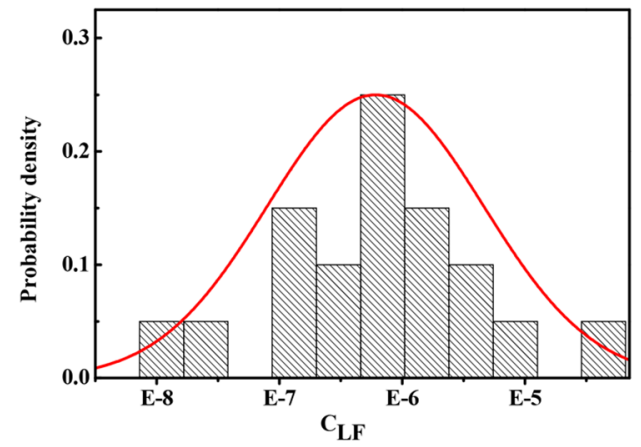

(b)

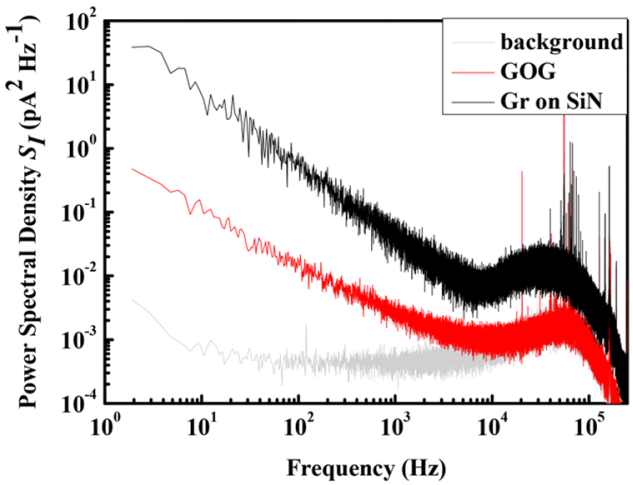

(d)

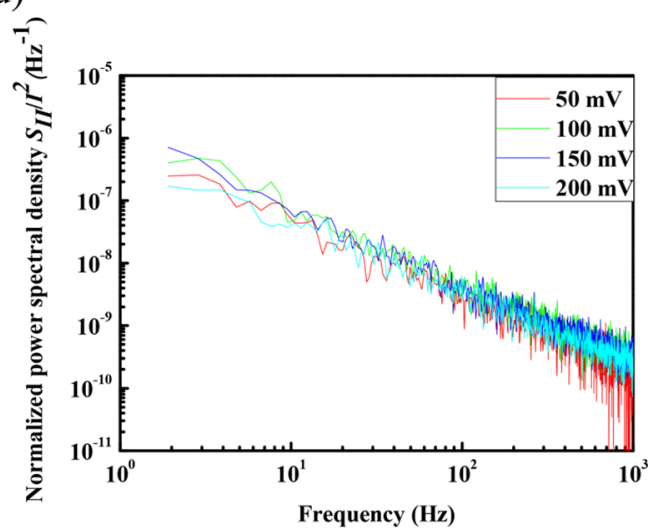

(f)

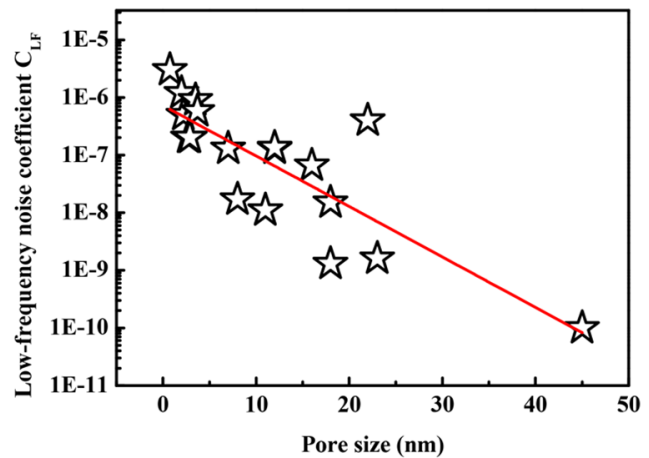

Figure 5. $1 / f$ noise of GOG nanopore. (a) Typical current trace of a GOG nanopore $(R=23 \mathrm{M} \Omega, d=4.8 \mathrm{~nm}$ in red) and of a graphene nanopore on $\mathrm{SiN}$ pore $(R=30 \mathrm{M} \Omega, d=3.8 \mathrm{~nm}$ in black) under $200 \mathrm{mV}$. (b) Spectral densities of the GOG nanopore and of a graphene nanopore on SiN pore. (c) Spectral densities $\left(S_{\mathrm{I}}\right)$ at various bias voltages for the GOG nanopore over the $1 / f$ regimes (for graphene pores this band is $1-1000 \mathrm{~Hz}$ ). (d) Normalized spectral densities $\left(S_{\mathrm{I}} / I^{2}\right)$ of the curves in c. A linear fit of these curves yields the low-frequency noise coefficient $C_{\mathrm{LF}}$ that represents the magnitude of the $1 / f$ noise. (e) Probability distributions of low-frequency noise coefficients $C_{\mathrm{LF}}$ of 19 GOG nanopores. (f) $C_{\mathrm{LF}}$ versus pore diameter was plotted logarithmically. The red line represents a linear fit of the data.

collected good statistics on 4 devices with pore diameters around $20 \mathrm{~nm}$. Here we present a typical experiment of DNA translocation, confirming the translocation through a single GOG nanopore, since DNA translocation in multiple pores will affect the conductance much more substantially (larger reductions than $3 \mathrm{nS}$ in the ionic conductance when more than one DNA translocates the nanopores at the same time). The formation of such GOG nanopores during the electrochemical etching process is likely initiated via a fluctuation that randomly selects an active site for the nanopore nucleation and is then limited to that site during the GOG nanopore formation and enlargement. The average translocation time is
$2.3 \pm 0.9 \mathrm{~ms}$ for the $48.5 \mathrm{~kb}$ double-stranded $\lambda$-DNA (Figure $4 \mathrm{c})$, corresponding to $0.05 \mu \mathrm{s} /$ base. This translocation speed is comparable to the previously reported translocation time on $\lambda$ DNA in graphene nanopores. ${ }^{2}$

Minimizing noise is of key importance to improve the minimum change in current that can be reliably detected at a given bandwidth. Generally, the noise spectrum in nanopore systems can be divided into a low-frequency regime $(f<\sim 1$ $\mathrm{kHz})$ and a high-frequency regime $(f>\sim 1 \mathrm{kHz})$. Whereas the low-frequency noise is dominated by pink noise with a $1 / f$ dependence, $^{23}$ the high-frequency noise originates from the 
parasitic capacitance ${ }^{24}$ and prevents sampling at highfrequency bandwidths.

Figure 5a provides the typical ionic current traces for a GOG nanopore in red and a SiN membrane supported graphene nanopore in black, respectively. Both graphene nanopores were suspended over a pore of $1 \mu \mathrm{m}$ diameter. Both traces presented in Figure 5a were recorded at $200 \mathrm{mV}$ bias voltage and processed following the same protocol (low-pass filtered with an 8-pole Besssel filter at $100 \mathrm{kHz}$ ). Strikingly, the GOG nanopore exhibits much lower current noise than its counterparts on the $\mathrm{SiN}$ membrane window. In Figure 5b, the corresponding noise spectra reveals one order of magnitude lower noise for the GOG nanopore than the SiNsupported graphene nanopore. At the high-frequency region, the reduction of noise in the GOG nanopores can be attributed to the fact that a SiN membrane of tens of nanometers in thickness is replaced by a thick glass substrate (thickness of $\sim 100 \mu \mathrm{m}$ ) with low parasitic capacitance, which significantly reduces the capacitance of the whole support and resulting in consistently reduced electrical noise. ${ }^{25}$

Similarly, in the low-frequency regime, the amplitude of $1 / f$ noise for the GOG nanopore is also around one order of magnitude lower than that for graphene nanopores on $\mathrm{SiN}$ (Figure $5 b$ ). It is well-known that the $1 / f$ noise of graphene nanopores fabricated on the $\mathrm{Si}$ supported $\mathrm{SiN}$ membrane is relatively large and is typically two orders of magnitude higher than for silicon nitride nanopores. ${ }^{26}$ This large low-frequency $1 / f$ noise is most probably due to either surface contamination introduced during preparation/storage or mechanical fluctuations of the free-standing graphene membrane in aqueous solution. $^{26}$

Power spectral density $S_{\mathrm{I}}$ at varying voltage levels for a given graphene nanopore shows that $S_{\mathrm{I}}$ curves are bias-voltage dependent (Figure 5c). Indeed, the normalized power spectral densities-found by dividing the current power spectral density $S_{\text {I }}$ by the squared current amplitude, $\left(S_{\mathrm{I}}\right) / I^{2}$ - exhibit the same low-frequency noise magnitude and show no biasvoltage dependency (Figure 5d). In order to compare the noise levels for different samples under various bias conditions, we adopted $\frac{S_{\mathrm{I}}}{I^{2}}=\frac{C_{\mathrm{LF}}}{f}$, where $C_{\mathrm{LF}}$ represents the low-frequency noise amplitude. Here, based on the normalized power spectral density measurement (Figure 5d) on a total of 19 GOG chips, the histogram of $C_{\mathrm{LF}}$ (Figure $5 \mathrm{e}$ ) indicates that the low frequency $1 / f$ noise of the GOG nanopore is statistically one order of magnitude lower than that of electrically fabricated or TEM drilled graphene nanopores on $\mathrm{SiN}$ membrane in other studies. That is, the red curves depict log-normal distributions exhibiting an average value of $\left\langle C_{\mathrm{LF}}\right\rangle=5.8 \times 10^{-7}$ for GOG nanopores, compared to $\left\langle C_{\mathrm{LF}}\right\rangle=6.3 \times 10^{-6}$ for previously reported graphene nanopores in $\mathrm{SiN}$ membranes. ${ }^{26}$ We ascribe such exceptionally low $1 / f$ noise to the ultraclean nature of the electrochemically formed nanopores, as well as to the removal of thermal vibration from the solid support of the glass substrate compared to the free-standing $\mathrm{SiN}$ membrane. It was reported that as a nanoscale-microscale electromechanical mixing system, the graphene resonator was able to transduce the motion of the coupled free-standing metal electrode with minimal damping. ${ }^{27,28}$ We also investigated the dependence of the $1 / f$ noise on the size of the graphene pores. Figure $5 f$ plots the $1 / f$ noise as a function of pore diameter. The pore diameters are determined from conductance calculation based on the assumption that the resistance of graphene nanopore is dominated by the access resistance $\mathrm{R}=\sigma^{-1} \frac{1}{d}$, where $\sigma$ represents the bulk conductivity and $d$ is the pore diameter. ${ }^{26}$ Although there is appreciable scatter in the data, we evidenced a correlation between the size and the amplitude of the $1 / f$ noise where large pores have lower noise, in accordance with previous studies on $\mathrm{SiN}$ membrane supported graphene nanopores. ${ }^{26}$ Thus, our results clearly demonstrate order-ofmagnitude reduced current noise in electrochemically formed GOG nanopores, which yields ultraclean nanopores with a favorable reduction in the parasitic capacitive coupling and in the mechanical vibration coupling, making our approach highly promising for potential practical application of graphene nanopores for single molecule detection.

\section{CONCLUSION}

We unraveled the mechanism of ultraclean graphene nanopore/nanogap formation via electrochemical etching based on a graphene-on-glass platform. We conducted in situ liquid AFM studies and demonstrated the enlargement of a graphene nanostructure taking place at its freestanding edges during electrical fabrication. This electrochemical mechanism is strongly supported by our observed electrical etching of CVD graphene on silicon substrate under similar electrochemical conditions. One step further, we confirmed the electrochemical formation of graphene nanopores by doublestranded DNA translocation experiments and envision that this electrochemical etching mechanism offers a new strategy to meet the challenges of ultraclean graphene device fabrication at nanometer scale, including nanogaps. Particularly, the ultraclean GOG nanopores with exceptionally low noise level and ease of fabrication under voltage pulse make this a versatile platform to be used for improved nanopore measurement. Furthermore, such a platform also featured as a relatively cheap substrate to obtain nanopores in various $2 \mathrm{D}$ materials for various nanoelectronics and biomolecule detection applications.

\section{ASSOCIATED CONTENT}

\section{Supporting Information}

The Supporting Information is available free of charge at https://pubs.acs.org/doi/10.1021/acssensors.0c00883.

In situ liquid AFM system; Experimentally measured parasitic capacitance; Stability of GOG nanopores; Circuit diagram of a suspended graphene membrane on a $\mathrm{SiN}$ membrane; Polycrystalline CVD graphene with micrometer-sized grains; DNA translocation under different applied voltage (PDF)

\section{AUTHOR INFORMATION}

\section{Corresponding Authors}

Wangyang Fu - School of Materials Science and Engineering, Tsinghua University, 100084 Beijing, China; (1) orcid.org/ 0000-0003-3185-0457; Email: fwy2018@

mail.tsinghua.edu.cn

Grégory F. Schneider - Leiden Institute of Chemistry, Leiden University, 2333 CC Leiden, Netherlands; 이이.org/00000001-5018-3309; Email: g.f.schneider@chem.leidenuniv.nl

\section{Authors}

Xiaoyan Zhang - School of Materials Science and Engineering, Tsinghua University, 100084 Beijing, China; Leiden Institute of Chemistry, Leiden University, 2333 CC Leiden, Netherlands 
Pauline M. G. van Deursen - Leiden Institute of Chemistry, Leiden University, 2333 CC Leiden, Netherlands

Complete contact information is available at: https://pubs.acs.org/10.1021/acssensors.0c00883

\section{Notes}

The authors declare no competing financial interest.

\section{ACKNOWLEDGMENTS}

The authors acknowledge financial support of the European Research Council under the European Union's Seventh Framework Programme (FP/2007-2013)/ERC Grant Agreement no. 335879 project acronym "Biographene", The Netherlands Organization for Scientific Research (NWOVIDI 723.013.007, NWO-VENI 722.014.004), the Swiss National Science Foundation (SNSF P300P2_154557, P300P2 164663), European Commission Horizon 2020Research and Innovation Framework Programme (Marie Sklodowska-Curie actions Individual Fellowship No.749671) and National Natural Science Foundation of China.

\section{REFERENCES}

(1) Garaj, S.; Hubbard, W.; Reina, A.; Kong, J.; Branton, D.; Golovchenko, J. A. Graphene as a subnanometre trans-electrode membrane. Nature 2010, 467 (7312), 190-3.

(2) Schneider, G. F.; Kowalczyk, S. W.; Calado, V. E.; Pandraud, G.; Zandbergen, H. W.; Vandersypen, L. M.; Dekker, C. DNA translocation through graphene nanopores. Nano Lett. 2010, 10 (8), 3163-7.

(3) Merchant, C. A.; Healy, K.; Wanunu, M.; Ray, V.; Peterman, N.; Bartel, J.; Fischbein, M. D.; Venta, K.; Luo, Z.; Johnson, A. T.; Drndic, M. DNA translocation through graphene nanopores. Nano Lett. 2010, 10 (8), 2915-21.

(4) Heerema, S. J.; Dekker, C. Graphene nanodevices for DNA sequencing. Nat. Nanotechnol. 2016, 11 (2), 127-36.

(5) Schneider, G. F.; Dekker, C. DNA sequencing with nanopores. Nat. Biotechnol. 2012, 30 (4), 326-8.

(6) Arjmandi-Tash, H.; Belyaeva, L. A.; Schneider, G. F. Single molecule detection with graphene and other two-dimensional materials: nanopores and beyond. Chem. Soc. Rev. 2016, 45 (3), 476-93.

(7) Song, B.; Schneider, G. F.; Xu, Q.; Pandraud, G.; Dekker, C.; Zandbergen, $\mathrm{H}$. Atomic-scale electron-beam sculpting of near-defectfree graphene nanostructures. Nano Lett. 2011, 11 (6), 2247-50.

(8) Janssen, X. J.; Jonsson, M. P.; Plesa, C.; Soni, G. V.; Dekker, C.; Dekker, N. H. Rapid manufacturing of low-noise membranes for nanopore sensors by trans-chip illumination lithography. Nanotechnology 2012, 23 (47), 475302.

(9) Lee, M. H.; Kumar, A.; Park, K. B.; Cho, S. Y.; Kim, H. M.; Lim, M. C.; Kim, Y. R.; Kim, K. B. A low-noise solid-state nanopore platform based on a highly insulating substrate. Sci. Rep. 2015, 4, 7448.

(10) Kuan, A. T.; Lu, B.; Xie, P.; Szalay, T.; Golovchenko, J. A. Electrical pulse fabrication of graphene nanopores in electrolyte solution. Appl. Phys. Lett. 2015, 106 (20), 203109.

(11) Liang, X.; Sperling, B. A.; Calizo, I.; Cheng, G.; Hacker, C. A.; Zhang, Q.; Obeng, Y.; Yan, K.; Peng, H.; Li, Q.; Zhu, X.; Yuan, H.; Hight Walker, A. R.; Liu, Z.; Peng, L.-m.; Richter, C. A. Toward Clean and Crackless Transfer of Graphene. ACS Nano 2011, 5 (11), 91449153.

(12) Matsui, K.; Yanagi, I.; Goto, Y.; Takeda, K. Prevention of Dielectric Breakdown of Nanopore Membranes by Charge Neutralization. Sci. Rep. 2015, 5, 17819.

(13) Kwok, H.; Briggs, K.; Tabard-Cossa, V. Nanopore fabrication by controlled dielectric breakdown. PLoS One 2014, 9 (3), No. e92880.
(14) Yates, D. E.; Levine, S.; Healy, T. W. Site-binding Model of the Electrical Double Layer at the Oxide/ Water interface. J. Chem. Soc., Faraday Trans. 1 1974, 70 (0), 1807-1818.

(15) Laborde, C.; Pittino, F.; Verhoeven, H. A.; Lemay, S. G.; Selmi, L.; Jongsma, M. A.; Widdershoven, F. P. Real-time imaging of microparticles and living cells with CMOS nanocapacitor arrays. Nat. Nanotechnol. 2015, 10 (9), 791-5.

(16) Kulkarni, G. S.; Zhong, Z. Detection beyond the Debye screening length in a high-frequency nanoelectronic biosensor. Nano Lett. 2012, 12 (2), 719-23.

(17) Fu, W.; Feng, L.; Panaitov, G.; Kireev, D.; Mayer, D.; Offenhäusser, A.; Krause, H. J. Biosensing near the neutrality point of graphene. Sci. Adv. 2017, 3, No. e1701247.

(18) Ryu, G. H.; Lee, J.; Kang, D.; Jo, H. J.; Shin, H. S.; Lee, Z. Effects of dry oxidation treatments on monolayer graphene. 2D Mater. 2017, 4 (2), 024011.

(19) Fang, H.-T.; Liu, C.-G.; Liu, C.; Li, F.; Liu, M.; Cheng, H.-M. Purification of Single-Wall Carbon Nanotubes by Electrochemical Oxidation. Chem. Mater. 2004, 16 (26), 5744-5750.

(20) Schoenfisch, M. H.; Ross, A. M.; Pemberton, J. E. Electrochemical Cleaning of Surface-Confined Carbon Contamination in Self-Assembled Monolayers on Polycrystalline Ag and Au. Langmuir 2000, 16 (6), 2907-2914.

(21) Knopfmacher, O.; Tarasov, A.; Fu, W.; Wipf, M.; Niesen, B.; Calame, M.; Schonenberger, C. Nernst limit in dual-gated Sinanowire FET sensors. Nano Lett. 2010, 10 (6), 2268-74.

(22) Siwy, Z.; Fulinski, A. Origin of $1 / \mathrm{f}(\mathrm{alpha})$ noise in membrane channel currents. Phys. Rev. Lett. 2002, 89 (15), 158101.

(23) Smeets, R. M.; Keyser, U. F.; Dekker, N. H.; Dekker, C. Noise in solid-state nanopores. Proc. Natl. Acad. Sci. U. S. A. 2008, 105 (2), $417-21$.

(24) Rosenstein, J. K.; Wanunu, M.; Merchant, C. A.; Drndic, M.; Shepard, K. L. Integrated nanopore sensing platform with submicrosecond temporal resolution. Nat. Methods 2012, 9 (5), 487-92.

(25) Balan, A.; Chien, C. C.; Engelke, R.; Drndic, M. Suspended Solid-state Membranes on Glass Chips with Sub 1-pF Capacitance for Biomolecule Sensing Applications. Sci. Rep. 2016, 5, 17775.

(26) Heerema, S. J.; Schneider, G. F.; Rozemuller, M.; Vicarelli, L.; Zandbergen, H. W.; Dekker, C. $1 / \mathrm{f}$ noise in graphene nanopores. Nanotechnology 2015, 26 (7), 074001.

(27) Chen, C.; Rosenblatt, S.; Bolotin, K. I.; Kalb, W.; Kim, P.; Kymissis, I.; Stormer, H. L.; Heinz, T. F.; Hone, J. Performance of monolayer graphene nanomechanical resonators with electrical readout. Nat. Nanotechnol. 2009, 4 (12), 861-7.

(28) De Alba, R.; Massel, F.; Storch, I. R.; Abhilash, T. S.; Hui, A.; McEuen, P. L.; Craighead, H. G.; Parpia, J. M. Tunable phonon-cavity coupling in graphene membranes. Nat. Nanotechnol. 2016, 11 (9), 741-6.

(29) Plesa, C.; Dekker, C. Data analysis methods for solid-state nanopores. Nanotechnology 2015, 26 (8), 084003. 